\title{
Impact of multidisciplinary engagement in a quality improvement blood conservation protocol for craniosynostosis
}

\author{
David E. Kurlander, MD,1,3 Mona Ascha, MD, ${ }^{1,3}$ Danielle C. Marshall, MD, ${ }^{8}$ Derek Wang, MD, ${ }^{1}$ \\ Mustafa S. Ascha, MS, PhD, ${ }^{1}$ Paul A. Tripi, MD,,4 Hollie M. Reeves, D0,1,5 \\ Katharine A. Downes, MD, ${ }^{1,5}$ Sanjay Ahuja, MD, ${ }^{1,5}$ Alexandre T. Rotta, MD, ${ }^{7}$ \\ Gregory E. Lakin, MD, ${ }^{6}$ and Krystal L. Tomei, MD, MPH ${ }^{1,2}$
}

\begin{abstract}
${ }^{1}$ Case Western Reserve University, Cleveland; Departments of ${ }^{2}$ Neurological Surgery, ${ }^{3}$ Plastic Surgery, ${ }^{4}$ Anesthesiology, and ${ }^{5}$ Pediatrics, University Hospitals Rainbow Babies \& Children's Hospital, Cleveland, Ohio; ${ }^{6}$ South Florida Center for Cosmetic Surgery, Fort Lauderdale, Florida; ${ }^{7}$ Department of Pediatrics, Duke University, Durham, North Carolina; and ${ }^{8}$ Department of Orthopedic Surgery, University of Miami Hospital, Miami, Florida
\end{abstract}

OBJECTIVE Patients undergoing open cranial vault remodeling for craniosynostosis frequently experience substantial blood loss requiring blood transfusion. Multiple reports in the literature have evaluated the impact of individual blood conservation techniques on blood transfusion rates during craniosynostosis surgery. The authors engaged a multidisciplinary team and assessed the impact of input from multiple stakeholders on the evolution of a comprehensive quality improvement protocol aimed at reducing or eliminating blood transfusion in patients undergoing open surgery for craniosynostosis.

METHODS Over a 4-year period from 2012 to 2016, 39 nonsyndromic patients were operated on by a single craniofacial plastic surgeon. Initially, no clear blood conservation protocol existed, and specific interventions were individually driven. In 2014, a new pediatric neurosurgeon joined the craniofacial team, and additional stakeholders in anesthesiology, transfusion medicine, critical care, and hematology were brought together to evaluate opportunities for developing a comprehensive blood conservation protocol. The initial version of the protocol involved the standardized administration of intraoperative aminocaproic acid (ACA) and the use of a cell saver. In the second version of the protocol, the team implemented the preoperative use of erythropoietin (EPO). In addition, intraoperative and postoperative resuscitation and transfusion guidelines were more clearly defined. The primary outcomes of estimated blood loss (EBL), transfusion rate, and intraoperative transfusion volume were analyzed. The secondary impact of multidisciplinary stakeholder input was inferred by trends in the data obtained with the implementation of the partial and full protocols.

RESULTS Implementing the full quality improvement protocol resulted in a $66 \%$ transfusion-free rate at the time of discharge compared to $0 \%$ without any conservation protocol and $27 \%$ with the intermediate protocol. The administration of EPO significantly increased starting hemoglobin/hematocrit $(11.1 \mathrm{~g} / \mathrm{d} / 31.8 \%$ to $14.7 \mathrm{~g} / \mathrm{dl} / 45.6 \%, \mathrm{p}<0.05)$. The group of patients receiving ACA had lower intraoperative EBL than those not receiving ACA, and trends in the final-protocol cohort, which had received both preoperative EPO and intraoperative ACA, demonstrated decreasing transfusion volumes, though the decrease did not reach statistical significance.

CONCLUSIONS Patients undergoing open calvarial vault remodeling procedures benefit from the input of a multidisciplinary stakeholder group in blood conservation protocols. Further research into comprehensive protocols for blood conservation may benefit from input from the full surgical team (plastic surgery, neurosurgery, anesthesiology) as well as additional pediatric subspecialty stakeholders including transfusion medicine, critical care, and hematology.

https://thejns.org/doi/abs/10.3171/2020.4.PEDS19633

KEYWORDS craniosynostosis; blood conservation; calvarial vault remodeling; craniofacial

ABBREVIATIONS ACA = aminocaproic acid; $C V R=$ cranial vault remodeling; $E B L=$ estimated blood loss; $E P O=$ erythropoietin; $H b=$ hemoglobin; $\mathrm{Hct}=$ hematocrit. SUBMITTED October 21, 2019. ACCEPTED April 9, 2020.

INCLUDE WHEN CITING Published online June 12, 2020; DOI: 10.3171/2020.4.PEDS19633. 
$\mathrm{P}$ ATIENTS undergoing open cranial vault remodeling (CVR) for craniosynostosis frequently require blood transfusion because of high blood loss. ${ }^{1-5}$ In a recent study by the Pediatric Craniofacial Collaborative Group, nearly all patients younger than 24 months (91\%) and the majority of patients older than 24 months (76\%) who were undergoing CVR were transfused intraoperatively. ${ }^{4}$ Serious complications of blood transfusion include acute hemolytic reaction, exposure to infectious agents, transfusion-related acute lung injury (TRALI), and hyperkalemia from rapid infusion of stored blood. ${ }^{1,3,4,6-8}$ Largevolume transfusion may also increase the risk of complication and length of hospitalization. ${ }^{9}$

A variety of protocols and techniques have been proposed to reduce perioperative blood transfusion. ${ }^{3-5,10}$ Currently, no evidence-based best-practice guidelines exist, resulting in wide variability in practice patterns. This state was underscored by a recent Pediatric Craniofacial Collaborative Group publication. ${ }^{4}$

We convened an interdisciplinary stakeholder group consisting of individuals from plastic surgery, neurosurgery, transfusion medicine, anesthesiology, pediatric intensive care, and hematology to discuss options for reducing perioperative blood transfusions in patients undergoing open calvarial vault remodeling with the goal of both reducing the volume of transfusions and eliminating the need for transfusions when possible. Using a combination of literature review and expert opinion, we developed and implemented an intervention-based blood conservation protocol for these patients, which included the administration of preoperative erythropoietin (EPO) and intraoperative aminocaproic acid (ACA). In addition to these medical interventions, the interdisciplinary group aimed to identify other variables that could be standardized. We worked to standardize intraoperative fluid resuscitation and intraoperative and perioperative transfusion thresholds, raise awareness of intraoperative hemostasis, and increase knowledge and understanding of intraoperative and perioperative care among all members of the perioperative care team. The purpose of the present study was to evaluate the efficacy of this quality improvement protocol in nonsyndromic patients undergoing open CVR compared to a preprotocol cohort. All patients included in this retrospective study were within a single plastic surgeon's practice. Prior to convening the multidisciplinary group, the surgeon had worked with our anesthesiology team to implement intraoperative ACA infusion to reduce blood loss. Later, the multidisciplinary team discussed the additional strategies noted above based on additional available literature on EPO and the best practices shared by our transfusion medicine colleagues. Given the natural evolution of the protocol over time, three groups could be easily differentiated: group 1, which underwent surgery prior to any intervention; group 2 , which underwent surgery utilizing only intraoperative ACA; and group 3, which underwent surgery after full protocol implementation including preoperative EPO, intraoperative ACA, and perioperative resuscitation parameters and transfusion thresholds. We hypothesized that implementing this protocol would significantly decrease intraoperative estimated blood loss (EBL), decrease transfusion volumes, and increase the in- cidence of transfusion-free hospitalization. In our study, we evaluated the impact of the quality improvement protocol created by a multidisciplinary stakeholder group and aimed at reducing or eliminating blood transfusion in patients undergoing open calvarial vault remodeling.

\section{Methods \\ Study Design}

Approval from the institutional review board of Case Western Reserve University and University Hospitals was obtained for this retrospective cohort study. All patients who had undergone CVR performed by the senior plastic surgeon (G.E.L.) from 2012 to 2016 were identified. Those who had undergone open CVR for single-suture synostosis were included in our analysis. Patients were excluded if they had a syndromic diagnosis because of the greater blood loss expected in this group. ${ }^{11}$ Patients with coronal and metopic suture synostosis underwent frontoorbital advancement. Patients with sagittal suture synostosis underwent calvarial vault remodeling utilizing a reverse-pi technique.

Patients were divided into three groups based on the evolution of interventions aimed at reducing transfusions: group 1, preprotocol and did not receive ACA; group 2, preprotocol and did receive ACA; and group 3, full protocol and received EPO and ACA. Primary outcome variables were transfusion-free hospitalization rate, EBL, and intraoperative transfusion volume. Total transfusion volume, defined as intraoperative plus postoperative transfusion volume, was also calculated. EBL was determined immediately postoperatively after discussion between the surgeons and anesthesiologist. This estimate was based on the volume in the suction container and sponge saturation, which were not specifically weighed. Demographics and other intraoperative and perioperative data were recorded.

\section{Patient Protocol}

All included patients were from a single craniofacial plastic surgeon's practice. Early in the series, surgical collaboration for open CVR cases existed between two neurosurgeons. In mid-2014, a pediatric neurosurgeon joined the craniofacial team, and all surgeries from that point on were joint cases between the pediatric neurosurgeon and the craniofacial surgeon. Data collection was terminated in 2016 given the departure of the plastic surgeon from the institution, and a gap in the availability of a pediatric craniofacial surgeon precluded further patient inclusion. Early in the series (groups 1 and 2), no specific protocol was followed. Individual anesthesiologists drove decisions about the use of ACA, which was highly variable. ACA was chosen based on our anesthesia group's familiarity with this agent for use in spinal fusion procedures. ${ }^{12}$ There were no specific volume resuscitation or transfusion guidelines. Blood was not routinely transfused on initial incision. A cell saver was used when possible before and after protocol implementation. Later in the series, a team was formed to develop and implement a protocol to consistently care for patients undergoing open CVR, including specialists from pediatric plastic surgery, pediatric neurosurgery, pediatric anesthesiology, pediatric hematol- 
ogy/oncology, transfusion medicine, and pediatric critical care. Protocol development included consideration of preoperative optimization, intraoperative interventions, and transfusion thresholds that were generated with input from the entire team and were based on a review of the literature and expert opinion.

Our specific blood conservation interventions are delineated in Table 1 . The preoperative protocol included a pediatric hematology consultation 6 weeks prior to surgery for evaluation for Epogen administration. Intraoperatively, patients received ACA at a loading dose of $100 \mathrm{mg} /$ $\mathrm{kg}$ over 1 hour starting after induction of anesthesia and a maintenance dose of $10 \mathrm{mg} / \mathrm{kg}$, which was discontinued at the completion of surgery. Blood loss replacement was standardized and specific thresholds for transfusion were established. No specific volume of intravenous fluids was delineated to allow for accommodation of fluid deficits, length of fasting, urine output, and patient response to resuscitation; however, the type and order of fluid replacement was standardized to reduce bias between providers. Intraoperative blood loss was based on an estimate in the suction containers and sponge and drape saturation, and a volume was agreed on by both participating surgeons and the anesthesiologist. All patients were transferred to the pediatric ICU postoperatively and were typically transferred to the regular surgical nursing floor on postoperative day 1. A postoperative protocol for blood draws and transfusion thresholds was standardized.

\section{Statistical Analysis}

Descriptive statistics were calculated for clinical and demographic characteristics. The Tukey honestly significant difference (HSD) multiple comparison was performed to test for differences in age, weight, and duration of surgery across protocol groups. The independence of proportions of patients across sex and cranial sutures fused was determined using two-sided Fisher's exact tests. The main outcomes of interest, EBL and transfusion volume, were tested for differences across each of the protocol groups using the Tukey HSD.

Hematocrit (Hct) and hemoglobin $(\mathrm{Hb})$ differences were tested across the group of patients treated with EPO compared to those who did not receive EPO. For each protocol group, paired t-tests were used to ascertain differences between preoperative and postoperative values of $\mathrm{Hb}$ and Hct. Two-sided Wilcoxon rank-sum tests were performed to test for an increase in $\mathrm{Hb}$ and $\mathrm{Hct}$ in the population treated with EPO compared to the patients not treated with EPO. Among the patients not treated with EPO, twosided Wilcoxon rank-sum tests were performed to test for a difference in $\mathrm{Hb}$ and $\mathrm{Hct}$ across protocol groups.

Statistical significance was defined as $\mathrm{p}<0.05$; statistics are suppressed where 5 or fewer observations were available in a category, except when using Fisher's exact test. All analysis was performed in $\mathrm{R}$ version 3.4.0 ( $\mathrm{R}$ Foundation for Statistical Computing).

\section{Results}

Forty-one consecutive patients who had undergone CVR performed by a single plastic surgeon were identi-

\section{TABLE 1. Blood conservation protocol}

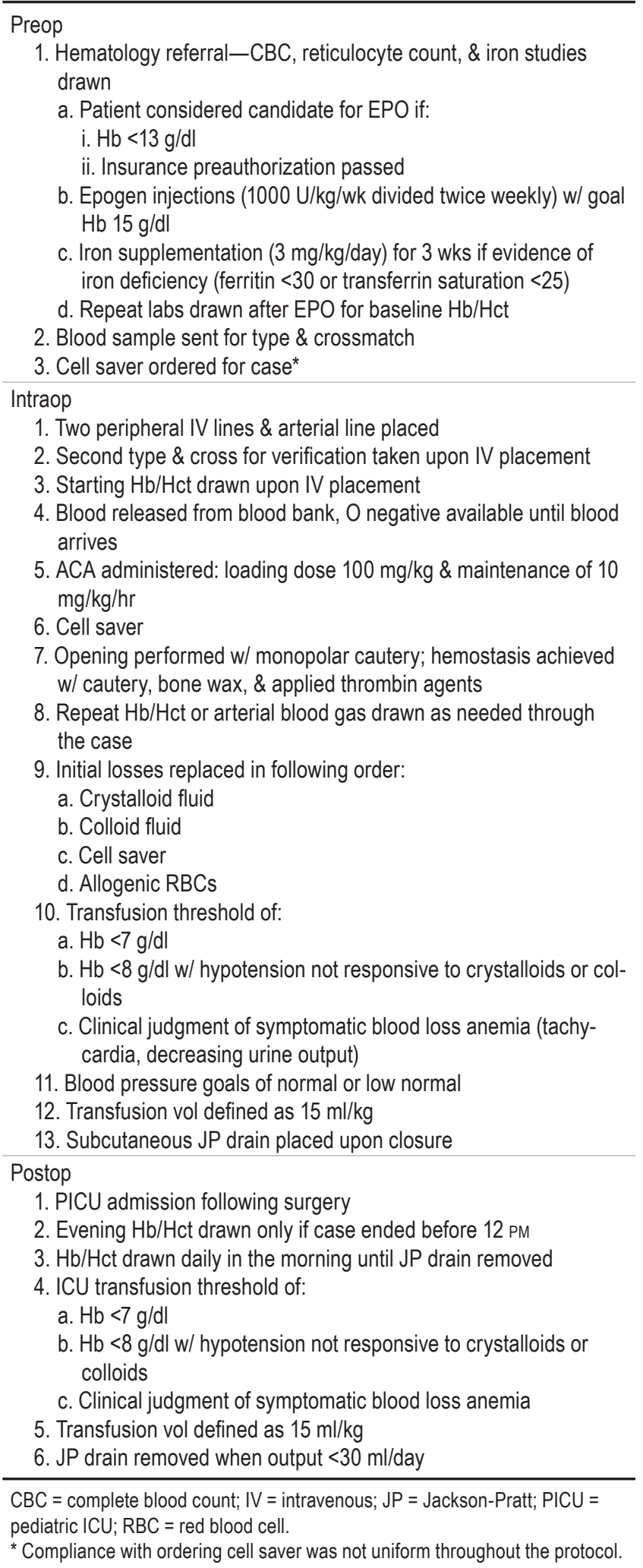

fied. After the exclusion of 2 syndromic patients, 39 patients were eligible for analysis: 19 patients in group 1, 11 patients in group 2, and 9 patients in group 3. 
TABLE 2. Patient characteristics across treatment groups

\begin{tabular}{lcccc}
\hline & Group 1 & Group 2 & Group 3 & p Value \\
\hline No. & 19 & 11 & 9 & \\
\hline Mean age in mos (SD) & $10.8(7.1)$ & $13.0(12.1)$ & $9.0(4.3)$ & 0.571 \\
\hline Mean weight in kg (SD) & $8.9(1.9)$ & $9.9(3.9)$ & $8.1(1.9)$ & 0.328 \\
\hline Suture fused, no. (\%) & & & \\
\hline Metopic & $9(47)$ & $2(18)$ & $2(22)$ & 0.190 \\
\hline Sagittal & $10(53)$ & $8(73)$ & $7(78)$ & 0.337 \\
\hline Coronal & $1(5)$ & $2(18)$ & $0(0)$ & 0.271 \\
\hline Lambdoid & $1(5)$ & $2(18)$ & $0(0)$ & $*$ \\
\hline Mean preop Hb/Hct in g/dl/\% & $11.4 / 33.5$ & $11.1 / 32.7$ & $13.3 / 41.4$ & $0.003 \dagger$ \\
\hline Mean duration of surgery in mins (SD) & $342(86)$ & $359(117)$ & $346(98)$ & 0.948 \\
\hline Mean length of hospitalization in days (SD) & $5.3(5.5)$ & $3.6(1.4)$ & $2.8(0.8)$ & 0.265 \\
\hline Cell saver transfusion, no. (\%) & $3(15.8)$ & $2(18.2)$ & $1(11.1)$ & 0.907 \\
\hline
\end{tabular}

* No $p$ value was presented as patients with unilateral and bilateral lambdoid suture synostosis were evaluated separately, and the table presents merged values.

$\dagger$ The preoperative $\mathrm{Hb} / \mathrm{Hct} \mathrm{p}$ value was obtained by comparing patients who had received EPO with those who had not, rather than among all three groups.

No statistically significant differences were found in age, weight, or duration of surgery across the protocol groups ( $\mathrm{p}>0.05$; Table 2). There were significant differences among the groups in terms of sex $(\mathrm{p}<0.05)$. Differences in the use of cell saver could not be assessed because of inadequate sample sizes. Group 3 had significantly higher starting $\mathrm{Hb}$ and $\mathrm{Hct}(\mathrm{Hb} / \mathrm{Hct})$ than the groups not receiving EPO $(\mathrm{p}<0.05)$. Mean $\mathrm{Hb} / \mathrm{Hct}$ increased from $11.1 \mathrm{~g} / \mathrm{dl} / 32.7 \%$ before EPO treatment to $14.7 \mathrm{~g} / \mathrm{dl} / 45.6 \%$ after treatment but before surgery $(\mathrm{p}<0.05)$. No adverse events relating to EPO or ACA were observed.

In group 3, 66\% of the patients were discharged transfusion free, compared to $27 \%$ in group 2 and $0 \%$ in group 1 ( $<<0.05$; Fig. 1). Groups 3 and 2 had statistically significantly lower EBL than group $1(11.7$ and $16.7 \mathrm{ml} / \mathrm{kg}$ vs $47.9 \mathrm{ml} / \mathrm{kg}$, respectively, $\mathrm{p}<0.05$; Fig. 2). There was no significant difference in EBL between groups 3 and 2 $(\mathrm{p}=0.82)$. Group 3 and group 2 required a significantly lower intraoperative transfusion volume than group 1 (1.0 and $17.1 \mathrm{ml} / \mathrm{kg}$ vs $46.7 \mathrm{ml} / \mathrm{kg}$, respectively, p < 0.05; Fig. $3)$. A lower intraoperative transfusion volume was given to group 3 than to group 2, but the difference between the two was not significant $(\mathrm{p}=0.49)$. Additionally, group 3

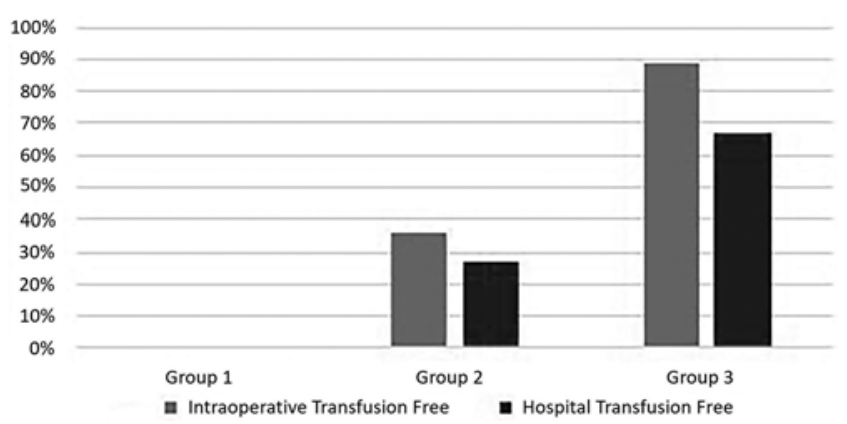

FIG. 1. Intraoperative and total in-hospital transfusion-free rates across protocol groups. and group 2 received a significantly lower total transfusion volume (intraoperative + postoperative transfusion) than group 1 ( 4.3 and $19.6 \mathrm{ml} / \mathrm{kg}$ vs $51.5 \mathrm{ml} / \mathrm{kg}, \mathrm{p}<0.05$ ). A lower total transfusion volume was required in group 3 than in group 2, but the difference between the two was not significant $(\mathrm{p}=0.52)$. Group 3 had the shortest hospital stay, followed by group 2 and group 1, although the differences were not significant ( 2.8 vs 3.6 vs 5.3 days, $\mathrm{p}>0.05$ ).

\section{Discussion}

There is extensive literature regarding the importance of blood transfusion protocols to reduce transfusions in pediatric patients undergoing calvarial vault remodeling surgery. However, the majority of these protocols have been developed with consideration from only a few specialties. Many have involved some combination of plastic surgery, neurosurgery, and anesthesia specialties or have been largely driven by a single specialty involved in this care..$^{3-5,10}$ In this study, we sought to evaluate the impact of multidisciplinary stakeholder input, including nonsurgical specialties, on a quality improvement-based blood conservation protocol for open CVR in the treatment of craniosynostosis. We hypothesized that this comprehensive protocol would improve outcomes compared to those in two preprotocol groups undergoing the same surgery, one receiving ACA and the other not receiving ACA.

The results of this study demonstrated that our comprehensive protocol, consisting of intervention with EPO and ACA as well as the standardization of perioperative and intraoperative resuscitation and transfusion thresholds, lowers the transfusion rate, EBL, and transfusion volumes compared to those in a preprotocol cohort. Compared to a preprotocol cohort that received ACA but without the other interventions or standardization, the patients on our protocol had similar EBL, an expected finding given that EPO has no impact on EBL. Patients on our protocol also had a decreased transfusion volume compared to the vol- 


\begin{tabular}{ccccc}
\hline & Difference & $2.5 \%$ & $97.5 \%$ & P Value \\
\hline Amicar-Neither & -31.21 & -48.13 & -14.29 & 0.0001931 \\
EPO and Amicar-Neither & -36.15 & -54.22 & -18.08 & $6.147 \mathrm{e}-05$ \\
EPO and Amicar-Amicar & -4.936 & -25.01 & 15.14 & 0.8204 \\
\hline
\end{tabular}

Estimated blood loss across treatment groups

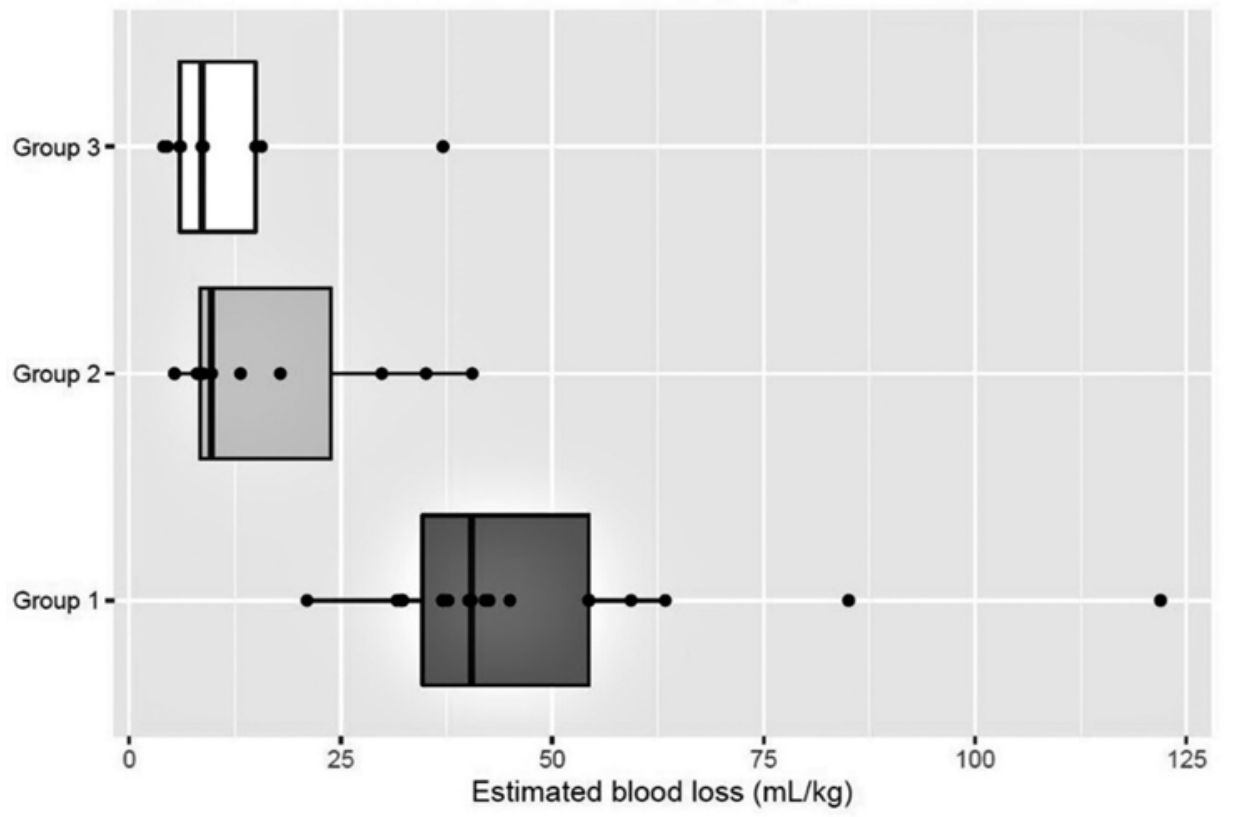

FIG. 2. EBL across protocol groups: group 1, preprotocol, no ACA; group 2, preprotocol with ACA; group 3, full protocol. AmicarNeither = group 2 versus group 1; EPO and Amicar-Amicar = group 3 versus group 2; EPO and Amicar-Neither = group 3 versus group 1.

ume in the preprotocol cohort that had received ACA, although the difference between the two was not statistically significant. Eighty-nine percent of patients in the finalprotocol group left the operating room transfusion free, and $66 \%$ were discharged from the hospital transfusion free. Based on the results of our study, a comprehensive, multifaceted approach to a blood conservation protocol that includes preoperative EPO, intraoperative antifibrinolytics, and guidelines on transfusion threshold and volume throughout the surgery and the postoperative period may decrease transfusion volume in patients undergoing open CVR. The additional input of teams not typically associated with developing blood conservation protocols informed our decision-making process. These teams included transfusion medicine, hematology, and pediatric intensive care.

Our specific active interventions included preoperative EPO and intraoperative ACA. One of the FDA-approved indications for Epogen is to reduce the need for allogenic transfusions, though in pediatric patients, this indication is not as well established. EPO is FDA approved for the reduction of allogenic blood transfusion in adult surgical patients expected to experience high blood loss. In patients 1 month to 2 years of age, EPO is FDA approved for anemia related to hemodialysis, chronic renal disease not requiring hemodialysis, $\mathrm{HIV}$, and chemotherapy. EPO is not explicitly FDA approved in pediatric patients undergoing surgery. ${ }^{13}$
However, antifibrinolytics have a long record of use in pediatric cardiac, general, and orthopedic surgery. ${ }^{14-24}$ The most commonly used antifibrinolytics, ACA and tranexamic acid, likely have similar efficacy ${ }^{25,26}$ and work by reversibly blocking the lysine-binding sites of plasminogen, preventing activation to plasmin and lysis of polymerized fibrin. A growing body of evidence supports the finding that antifibrinolytic administration minimizes transfusion requirements in patients undergoing open CVR. ${ }^{19,27,28}$ In randomized studies, Dadure et al. and Goobie et al. showed decreased transfusion volumes in patients undergoing CVR surgery who had received tranexamic acid. ${ }^{29,30}$ In addition to reduced transfusion requirements, Oppenheimer et al. reported that patients receiving ACA were less likely to require a second unit of blood, therefore reducing exposures to donor blood antigens. ${ }^{2}$ In a retrospective study, Hsu et al. reported decreased EBL, intraoperative donor exposure, and postoperative drain output with ACA. ${ }^{31}$

Thromboembolic events and seizures have been rarely reported in association with antifibrinolytic use in cranial and noncraniofacial surgery. ${ }^{22,28,32-34}$ However, in a multiinstitutional study including 1638 CVR cases from 31 institutions, Goobie et al. found no association between thromboembolic events or seizures and antifibrinolytic use. ${ }^{35} \mathrm{~A}$ systematic review by Basta et al. also supported the safety of antifibrinolytics. ${ }^{14}$ There may be an increased risk of sei- 


\begin{tabular}{ccccc}
\hline & Difference & $2.5 \%$ & $97.5 \%$ & P Value \\
\hline Amicar-Neither & -29.68 & -58.42 & -0.9379 & 0.04174 \\
EPO and Amicar-Neither & -45.75 & -76.45 & -15.06 & 0.002371 \\
EPO and Amicar-Amicar & -16.07 & -50.17 & 18.02 & 0.4889 \\
\hline
\end{tabular}

\section{Estimated transfusion volume}

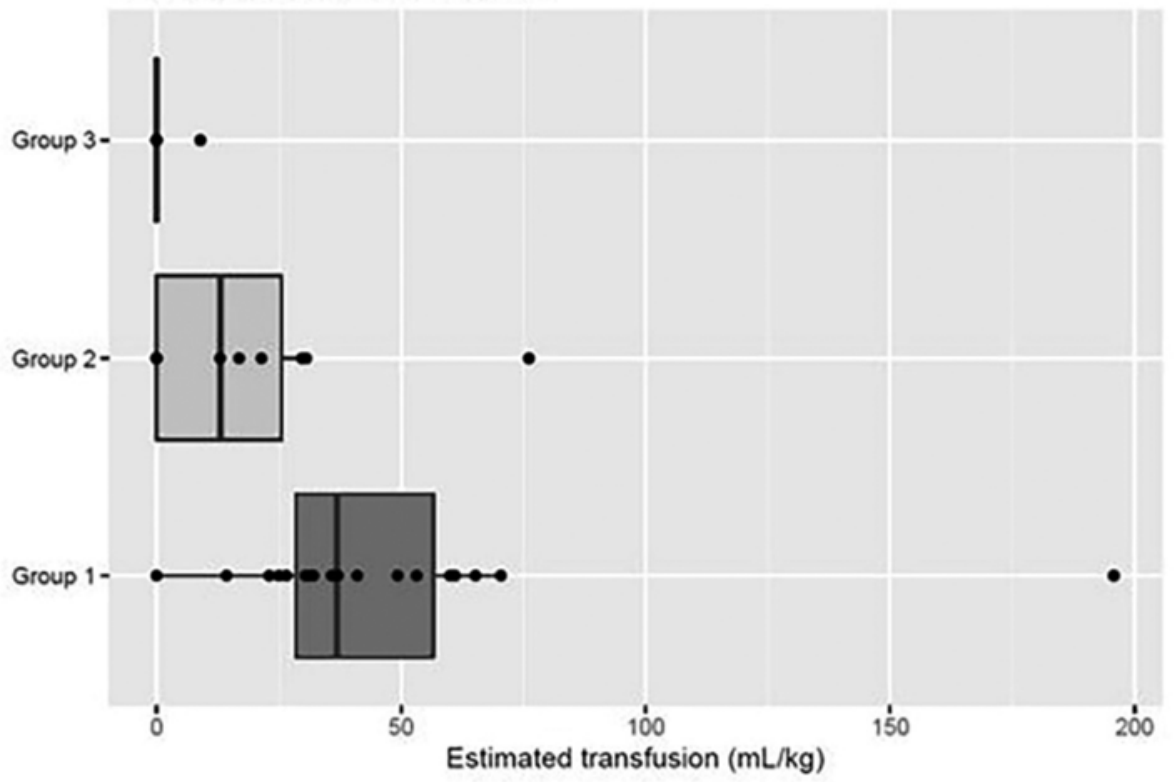

FIG. 3. Estimated intraoperative transfusion volume in milliliters per kilogram across protocol groups.

zure using tranexamic acid versus ACA and an increased risk of renal dysfunction in patients receiving ACA versus tranexamic acid, although evidence is limited. ${ }^{25,26}$ Despite evidence of their safety and efficacy, antifibrinolytics were administered to only $63 \%$ of children in the study by Stricker et al. ${ }^{4}$ Before protocol implementation at our institution, when antifibrinolytic use was driven by individual anesthesiologists and the institutional availability of ACA only, ACA was used in $37 \%$ of open CVR cases. We report no complications relating to ACA and are satisfied with the evidence supporting the safety of antifibrinolytics. However, in all patients with a history of seizures, hypercoagulable propensity, or renal disease, caution is advised.

Nguyen et al. evaluated a protocol developed among plastic surgery, neurosurgery, and anesthesiology specialties, which emphasized intraoperative ACA (bolus dose at $100 \mathrm{mg} / \mathrm{kg}$ over 30 minutes, then infused at $33 \mathrm{mg} / \mathrm{kg} /$ hr during surgery). ${ }^{10}$ In addition, they utilized thromboelastography (TEG) to determine the need for fresh frozen plasma (FFP) transfusion (infused preprotocol for a threshold international normalized ratio [INR] of 1.5). Implementation of their protocol decreased the volume of blood transfused from a mean intraoperative transfusion of $36.9 \mathrm{ml} / \mathrm{kg}$ to $19.2 \mathrm{ml} / \mathrm{kg}$. An evaluation of their postoperative transfusion volumes did not reveal a deference to higher transfusion volumes in the postoperative period (postoperative transfusion volumes of $26.7 \mathrm{ml} / \mathrm{kg}$ preprotocol and $20.2 \mathrm{ml} / \mathrm{kg}$ postprotocol). Overall, their cohort received blood from fewer discrete donors, fewer FFP transfusions, and a higher volume of crystalloid infusion postprotocol. They suggested that the protocol allowed tolerance of a lower $\mathrm{Hb}$ threshold prior to transfusion. Despite the lower transfusion volumes, $100 \%$ of patients in their series underwent allogenic transfusion both pre- and postprotocol.

The other active intervention in our protocol is EPO, which is supported by limited studies on CVR. Fearon and Weinthal reported a decreased transfusion rate and no adverse events in a randomized trial of EPO in 29 patients undergoing craniosynostosis repair. ${ }^{1}$ Meneghini et al. used a protocol consisting of preoperative EPO and preoperative acute normovolemic hemodilution for craniosynostosis and reported decreased transfusion requirements. ${ }^{36}$

Our protocol included other multidisciplinary-developed guidelines. We intended to use a cell saver device for blood recycling before and after protocol implementation. However, compliance with usage was limited. Overall, only 3 patients in group 1, 2 patients in group 2, and 1 patient in group 3 were noted to have a cell saver present intraoperatively, suggesting that this aspect of our protocol was not well accepted. One patient in each group did not have an adequate amount of blood collected via the cell saver to transfuse. Although our intent was to include the use of a cell saver in all surgeries postprotocol, because of the poor compliance with this aspect of our protocol, we are unable to assess the impact of this on transfusion rate or whether this may represent a cost-prohibitive adjuvant given the low volumes collected. The poor compliance likely stemmed from early experience with the cell saver device in which only a minimal volume of blood for transfusion was obtained, as well as from the cost of the device in comparison to the return. 
We also defined restrictive transfusion guidelines based on evidence in the pediatric ICU and pediatric surgery literature. ${ }^{37,38}$ It is likely that this contribution to our protocol had a significant impact, though we did not evaluate this as an independent variable. We noted in the review of our patients that, interestingly, although all of them had safe final $\mathrm{Hb} / \mathrm{Hct}$ before discharge, the patients in group 3 had lower $\mathrm{Hb} / \mathrm{Hct}$ than those in groups 1 and $2(8.8 \mathrm{~g} / \mathrm{dl} / 27.6 \%$ vs $9.1 \mathrm{~g} / \mathrm{dl} / 25.8 \%$ vs $10.6 \mathrm{~g} / \mathrm{dl} / 30.0 \%)$. It is likely that before protocol implementation, we were over-transfusing, which has associated risks and costs. Though this was not an independently analyzed variable, whether over-transfusing translated into unnecessary transfusion warrants further evaluation. Unfortunately, given the retrospective nature of our study, it would be difficult to evaluate what impact the total volume of transfusion for those over-transfused patients had on $\mathrm{Hb} / \mathrm{Hct}$ and thus whether transfusion could have been completely avoided in over-transfused patients. This result may have been attributable to a combination of factors including variable thresholds for transfusion between anesthesiologists and/or the intensive care team. In addition, establishing a clear understanding of the intraoperative preference for hemodilution allowed for improved tolerance of low postoperative $\mathrm{Hb}$ in asymptomatic patients. Since we were drawing serial labs, a low immediate postoperative $\mathrm{Hb}$ due to intended hemodilution may have resulted in transfusion of an asymptomatic patient prior to implementation of the full protocol.

Multiple other strategies exist for blood conservation. These are nicely outlined in a 2015 systematic review by White et al. ${ }^{5}$ Briefly, other techniques include the use of preoperative autologous donation, acute normovolemic hemodilution, drain reinfusion, rotational thromboeslastometry (ROTEM) or TEG, fibrin sealants, and epinephrine tumescence (C. Forrest, personal communication, 2017). ${ }^{1,3}$, 27,29,30,36,39-45 We are aware of several comprehensive blood transfusion protocols that have been reported in the literature and shown to be effective in decreasing the transfusion requirement. 4,10,47,48 However, many of these protocols have been established by a more limited team and are centered around intraoperative interventions, whereas our study expands the protocol to include pre- and postoperative care team members. The precise elements of an ideal protocol remain to be elucidated, with failed protocols less likely to be published. Our consideration of additional team members who are not directly involved in the intraoperative events allowed us to create a more robust protocol. This included thoughts about preoperative optimization with input from hematology and transfusion medicine and allowed our intensivists to better understand some of the intraoperative interventions that would impact their postoperative management and decision-making. Separately, an improved relationship with transfusion medicine allowed the surgeons to more effectively communicate to parents and families the risks of blood transfusion (from a singledonor transfusion as well as the added risks associated with blood donation from multiple donors) as well as serve as a point of contact for specific questions from families, including those interested in donor-directed transfusion options and risks.

\section{Study Limitations}

This study is limited by multiple factors inherent to a retrospective, single plastic surgeon design centered around a quality improvement protocol with a natural evolution of subgroups. As noted, the presence of any protocol has an impact on transfusion volume, and our protocol was implemented concurrently with a team-based discussion and standardization of the operative team to a single plastic surgeon and neurosurgeon pair. A learning curve for the new neurosurgeon could have decreased EBL over time; however, an evaluation of the EBL in sequential cases in group 1 (prior to any antifibrinolytic intervention and early in their practice) did not reveal any clear trend over time, suggesting that the protocol interventions were the primary contributing factors to the decrease in EBL and transfusion. Additionally, we evaluated the intragroup trends in EBL, and there was no significant progressive decrease within each group that would suggest an intragroup learning curve as a significant contribution to decreasing EBL. Moreover, EBL has been shown to underestimate calculated blood loss in CVR ${ }^{48,49}$ Certainly, the Hawthorne effect may be relevant to our study, as the mere implementation of the protocol as well as the addition of a new pediatric neurosurgeon may have impacted both the attention to hemostasis and the reported EBL, creating an observer bias. Our relatively small sample size allowed us to conclude significant differences in EBL between groups. However, it was not sufficiently powered to conclude that transfusion volumes were different between groups 2 and 3, which both received ACA, and ACA has already been proven to decrease transfusion rates in isolation, so the overall impact of the addition of EPO cannot be deduced.

We did note a difference in the relative frequency of fused sutures across the three groups, with an increased number of metopic synostosis cases in the earliest group. This may be attributable to a different frequency of referrals or a difference in the threshold for operative recommendation in milder cases or patients with a metopic ridge. Within-group comparisons of the sagittal vault cases versus frontoorbital advancements did not reveal a significant difference in EBL between the different surgical procedures. Considering the change in neurosurgeons during the study period, it is unclear whether there was any significant change in neurosurgical technique that may explain the differences in blood loss across the groups; however, the overall surgical procedure was largely unchanged. We did note that the addition of a pediatric neurosurgeon to the team likely contributed to the decrease in blood loss between group 1, for which the pediatric neurosurgeon was not present, and groups 2 and 3, for which a pediatric neurosurgeon was present. It is likely that a pediatric-trained neurosurgeon would be more observant of blood loss and intraoperatively address this loss in a more rapid manner, as evidenced by the observation that EBL in group 1 was largely impacted by three outliers. However, there was still a trend toward reduced blood loss and reduced transfusion in group 3, suggesting that attention to blood loss is only one component in the equation. In addition, once the surgical team was standardized, there were additional slight modifications to the surgery with an increasing number of 
barrel stave osteotomies in some of the later sagittal synostosis patients, which would have been expected to increase blood loss; however, this information was not specifically included in this analysis.

Finally, we did not attempt to determine the cost-effectiveness of the addition of EPO to our protocol. The addition of EPO required multiple preoperative visits as well as preauthorization of the medication and subsequent administration with follow-up laboratory values. Though this does not present a limitation to the analysis of our study, it is a potential limiting factor in a more widespread adoption of such a protocol.

\section{Conclusions}

The integration of additional subspecialties, such as transfusion medicine, intensive care, and hematology, may augment existing blood conservation protocols for nonsyndromic patients undergoing open CVR. Such a protocol at our institution decreased EBL and transfusion volume and increased the transfusion-free hospitalization rate compared to those in a preprotocol group. Active interventions such as EPO and ACA in combination with other blood conservation strategies may demonstrate additional benefit compared to ACA alone. Postoperative transfusion thresholds and the involvement of perioperative teams may reduce the risk of over-transfusing patients. Future studies will elucidate the elements of an ideal blood conservation protocol, factoring in efficacy, complications, and cost.

\section{References}

1. Fearon JA, Weinthal J. The use of recombinant erythropoietin in the reduction of blood transfusion rates in craniosynostosis repair in infants and children. Plast Reconstr Surg. 2002;109(7):2190-2196.

2. Oppenheimer AJ, Ranganathan K, Levi B, et al. Minimizing transfusions in primary cranial vault remodeling: the role of aminocaproic acid. J Craniofac Surg. 2014;25(1):82-86.

3. Stricker PA, Cladis FP, Fiadjoe JE, et al. Perioperative management of children undergoing craniofacial reconstruction surgery: a practice survey. Paediatr Anaesth. 2011;21(10):1026-1035.

4. Stricker PA, Goobie SM, Cladis FP, et al. Perioperative outcomes and management in pediatric complex cranial vault reconstruction: a multicenter study from the Pediatric Craniofacial Collaborative Group. Anesthesiology. 2017;126(2):276-287.

5. White N, Bayliss S, Moore D. Systematic review of interventions for minimizing perioperative blood transfusion for surgery for craniosynostosis. J Craniofac Surg. 2015;26(1):2636.

6. Buntain SG, Pabari M. Massive transfusion and hyperkalaemic cardiac arrest in craniofacial surgery in a child. Anaesth Intensive Care. 1999;27(5):530-533.

7. Lavoie J. Blood transfusion risks and alternative strategies in pediatric patients. Paediatr Anaesth. 2011;21(1):14-24.

8. Ririe DG, Lantz PE, Glazier SS, Argenta LC. Transfusionrelated acute lung injury in an infant during craniofacial surgery. Anesth Analg. 2005;101(4):1003-1006.

9. Chow I, Purnell CA, Gosain AK. Assessing the impact of blood loss in cranial vault remodeling: a risk assessment model using the 2012 to 2013 Pediatric National Surgical Quality Improvement Program data sets. Plast Reconstr Surg. 2015;136(6):1249-1260.

10. Nguyen TT, Hill S, Austin TM, et al. Use of blood-sparing surgical techniques and transfusion algorithms: association with decreased blood administration in children undergoing primary open craniosynostosis repair. J Neurosurg Pediatr. 2015;16(5):556-563.

11. Howe PW, Cooper MG. Blood loss and replacement for paediatric cranioplasty in Australia-a prospective national audit. Anaesth Intensive Care. 2012;40(1):107-113.

12. Florentino-Pineda I, Blakemore LC, Thompson GH, et al. The effect of $\varepsilon$-aminocaproic acid on perioperative blood loss in patients with idiopathic scoliosis undergoing posterior spinal fusion: a preliminary prospective study. Spine (Phila $P a$ 1976). 2001;26(10):1147-1151.

13. Procrit and Epogen. Package inserts. Amgen. Accessed April 22, 2020. https://www.accessdata.fda.gov/drugsatfda_docs/ label/2010/103234s5199lbl.pdf

14. Basta MN, Stricker PA, Taylor JA. A systematic review of the use of antifibrinolytic agents in pediatric surgery and implications for craniofacial use. Pediatr Surg Int. 2012;28(11):1059-1069.

15. Breuer T, Martin K, Wilhelm M, et al. The blood sparing effect and the safety of aprotinin compared to tranexamic acid in paediatric cardiac surgery. Eur J Cardiothorac Surg. 2009;35(1):167-171.

16. Brown JR, Birkmeyer NJO, O'Connor GT. Meta-analysis comparing the effectiveness and adverse outcomes of antifibrinolytic agents in cardiac surgery. Circulation. 2007;115(22):2801-2813.

17. Faraoni D, Goobie SM. The efficacy of antifibrinolytic drugs in children undergoing noncardiac surgery: a systematic review of the literature. Anesth Analg. 2014;118(3):628-636.

18. Henry DA, Moxey AJ, Carless PA, et al. Anti-fibrinolytic use for minimising perioperative allogeneic blood transfusion. Cochrane Database Syst Rev. 2001;(1):CD001886.

19. Henry D, Carless P, Fergusson D, Laupacis A. The safety of aprotinin and lysine-derived antifibrinolytic drugs in cardiac surgery: a meta-analysis. CMAJ. 2009;180(2):183-193.

20. Hutton B, Joseph L, Fergusson D, et al. Risks of harms using antifibrinolytics in cardiac surgery: systematic review and network meta-analysis of randomised and observational studies. BMJ. 2012;345:e5798.

21. Munoz JJ, Birkmeyer NJ, Birkmeyer JD, et al. Is epsilonaminocaproic acid as effective as aprotinin in reducing bleeding with cardiac surgery? A meta-analysis. Circulation. 1999;99(1):81-89.

22. Murkin JM, Falter F, Granton J, et al. High-dose tranexamic acid is associated with nonischemic clinical seizures in cardiac surgical patients. Anesth Analg. 2010;110(2):350-353.

23. Pasquali SK, Li JS, He X, et al. Comparative analysis of antifibrinolytic medications in pediatric heart surgery. $J$ Thorac Cardiovasc Surg. 2012;143(3):550-557.

24. Schouten ES, van de Pol AC, Schouten ANJ, et al. The effect of aprotinin, tranexamic acid, and aminocaproic acid on blood loss and use of blood products in major pediatric surgery: a meta-analysis. Pediatr Crit Care Med. 2009;10(2):182-190.

25. Martin K, Gertler R, Sterner A, et al. Comparison of bloodsparing efficacy of $\varepsilon$-aminocaproic acid and tranexamic acid in newborns undergoing cardiac surgery. Thorac Cardiovasc Surg. 2011;59(5):276-280.

26. Martin K, Breuer T, Gertler R, et al. Tranexamic acid versus $\varepsilon$-aminocaproic acid: efficacy and safety in paediatric cardiac surgery. Eur J Cardiothorac Surg. 2011;39(6):892-897.

27. Fearon JA. Reducing allogenic blood transfusions during pediatric cranial vault surgical procedures: a prospective analysis of blood recycling. Plast Reconstr Surg. 2004;113(4):1126-1130.

28. Hocker JR, Saving KL. Fatal aortic thrombosis in a neonate during infusion of epsilon-aminocaproic acid. J Pediatr Surg. 1995;30(10):1490-1492. 
29. Dadure C, Sauter M, Bringuier S, et al. Intraoperative tranexamic acid reduces blood transfusion in children undergoing craniosynostosis surgery: a randomized double-blind study. Anesthesiology. 2011;114(4):856-861.

30. Goobie SM, Meier PM, Pereira LM, et al. Efficacy of tranexamic acid in pediatric craniosynostosis surgery: a double-blind, placebo-controlled trial. Anesthesiology. 2011;114(4):862-871

31. Hsu G, Taylor JA, Fiadjoe JE, et al. Aminocaproic acid administration is associated with reduced perioperative blood loss and transfusion in pediatric craniofacial surgery. Acta Anaesthesiol Scand. 2016;60(2):158-165.

32. Lecker I, Wang D-S, Whissell PD, et al. Tranexamic acidassociated seizures: causes and treatment. Ann Neurol. 2016;79(1):18-26.

33. Martin K, Knorr J, Breuer T, et al. Seizures after open heart surgery: comparison of $\varepsilon$-aminocaproic acid and tranexamic acid. J Cardiothorac Vasc Anesth. 2011;25(1):20-25.

34. Sjoblom MD, Busso VO, Linscott LL, Sadhasivam S. Postoperative cerebral arterial thrombosis following cranial surgery in an infant. Paediatr Anaesth. 2013;23(11):1105-1106.

35. Goobie SM, Cladis FP, Glover CD, et al. Safety of antifibrinolytics in cranial vault reconstructive surgery: a report from the pediatric craniofacial collaborative group. Paediatr Anaesth. 2017;27(3):271-281. Published correction in Paediatr Anaesth. 2017;27(6):670.

36. Meneghini L, Zadra N, Aneloni V, et al. Erythropoietin therapy and acute preoperative normovolaemic haemodilution in infants undergoing craniosynostosis surgery. Paediatr Anaesth. 2003;13(5):392-396.

37. Lacroix J, Hébert PC, Hutchison JS, et al. Transfusion strategies for patients in pediatric intensive care units. $N$ Engl J Med. 2007;356(16):1609-1619.

38. Rouette J, Trottier H, Ducruet T, et al. Red blood cell transfusion threshold in postsurgical pediatric intensive care patients: a randomized clinical trial. Ann Surg. 2010;251(3):421-427.

39. Deva AK, Hopper RA, Landecker A, et al. The use of intraoperative autotransfusion during cranial vault remodeling for craniosynostosis. Plast Reconstr Surg. 2002;109(1):58-63.

40. Duncan C, Richardson D, May P, et al. Reducing blood loss in synostosis surgery: the Liverpool experience. J Craniofac Surg. 2008;19(5):1424-1430.

41. Haas T, Goobie S, Spielmann N, et al. Improvements in patient blood management for pediatric craniosynostosis surgery using a ROTEM ${ }^{\circledR}$-assisted strategy-feasibility and costs. Paediatr Anaesth. 2014;24(7):774-780.

42. Hans P, Collin V, Bonhomme V, et al. Evaluation of acute normovolemic hemodilution for surgical repair of craniosynostosis. J Neurosurg Anesthesiol. 2000;12(1):33-36.

43. Meara JG, Smith EM, Harshbarger RJ, et al. Blood-conservation techniques in craniofacial surgery. Ann Plast Surg. 2005;54(5):525-529.

44. Stehrer R, Hunger S, Schotten K-J, et al. Reduction of transfusion requirements in pediatric craniosynostosis surgery by a new local hemostatic agent. J Craniomaxillofac Surg. 2016;44(9):1246-1251.
45. Velardi F, Di Chirico A, Di Rocco C. Blood salvage in craniosynostosis surgery. Childs Nerv Syst. 1999;15(11-12):695710.

46. Vega RA, Lyon C, Kierce JF, et al. Minimizing transfusion requirements for children undergoing craniosynostosis repair: the CHoR protocol. $J$ Neurosurg Pediatr. 2014;14(2):190-195.

47. Velardi F, Di Chirico A, Di Rocco C, et al. "No allogeneic blood transfusion" protocol for the surgical correction of craniosynostoses. I. Rationale. Childs Nerv Syst. 1998;14(12):722-741.

48. Faberowski LW, Black S, Mickle JP. Blood loss and transfusion practice in the perioperative management of craniosynostosis repair. J Neurosurg Anesthesiol. 1999;11(3):167-172.

49. Seruya M, Oh AK, Boyajian MJ, et al. Unreliability of intraoperative estimated blood loss in extended sagittal synostectomies. J Neurosurg Pediatr. 2011;8(5):443-449.

\section{Disclosures}

Dr. Mustafa Ascha holds stock ownership in Roche and is an employee of Flatiron Health Inc., which is an independent subsidiary of the Roche Group.

\section{Author Contributions}

Conception and design: Tomei, Tripi, Reeves, Downes, Ahuja, Rotta, Lakin. Acquisition of data: Kurlander, Mona Ascha, Marshall, Wang. Analysis and interpretation of data: Kurlander, Mustafa Ascha, Lakin. Drafting the article: Tomei, Kurlander, Lakin. Critically revising the article: Tomei, Kurlander, Mona Ascha, Tripi, Rotta, Lakin. Reviewed submitted version of manuscript: all authors. Approved the final version of the manuscript on behalf of all authors: Tomei. Statistical analysis: Mustafa Ascha. Study supervision: Tomei, Lakin.

\section{Supplemental Information \\ Previous Presentations}

Portions of this work were presented in part at the American Society of Pediatric Neurosurgeons Annual Meeting held in Koloa, Hawaii, on January 10, 2019; the Ohio Valley Society of Plastic Surgeons Annual Meeting held in Pittsburgh, Pennsylvania, on June 4, 2017; and the American Society of Plastic Surgeons Annual Meeting held in Orlando, Florida, on October 9,2017

\section{Correspondence}

Krystal L. Tomei: Case Western Reserve University, Cleveland, OH. krystal.tomei@uhhospitals.org. 